\title{
Discovery of a Possible Early-T Thick-disk Subdwarf from the AllWISE2 Motion Survey*
}

\author{
Kendra Kellogg ${ }^{1,2}$ (1) , J. Davy Kirkpatrick ${ }^{2}$, Stanimir Metchev ${ }^{1,3}$ (1) Jonathan Gagné $e^{4,6}{ }^{(1)}$, and Jacqueline K. Faherty ${ }^{5}$ (1) \\ ${ }^{1}$ Department of Physics and Astronomy, Centre for Planetary and Space Exploration, The University of Western Ontario, \\ 1151 Richmond Street, London, ON N6A 3K7, Canada; kkellogg@uwo.ca \\ ${ }^{2}$ Infrared Processing and Analysis Center, Mail Code 100-22, California Institute of Technology, 1200 East California Boulevard, Pasadena, CA 91125, USA \\ ${ }^{3}$ Department of Physics and Astronomy, Stony Brook University, Stony Brook, NY, 11794-3800, USA \\ ${ }^{4}$ Carnegie Institution of Washington DTM, 5241 Broad Branch Road NW, Washington, DC 20015, USA \\ ${ }^{5}$ Department of Astrophysics, American Museum of Natural History, Central Park West at 79th Street, New York, NY 10034, USA \\ Received 2017 July 6; revised 2017 December 20; accepted 2017 December 21; published 2018 January 25
}

\begin{abstract}
We have discovered a potential T0 \pm 1 subdwarf from a search for sources in the AllWISE2 Motion Survey that do not have counterparts in surveys at shorter wavelengths. With a tangential velocity of $\sim 170 \mathrm{~km} \mathrm{~s}^{-1}$, this object-WISE J071121.36-573634.2 - has kinematics that are consistent with the thick-disk population of the Milky Way. Spectral fits suggest a low-metallicity for this object but also allow for the possibility of unresolved multiplicity. If WISE J0711-5736 is indeed an sdT0 dwarf, it would be only the second early-T subdwarf discovered to date.
\end{abstract}

Key words: brown dwarfs - infrared: stars - stars: individual (WISE J071121.36-573634.2) - stars: late-type stars: peculiar

\section{Introduction}

Brown dwarfs are the lowest-mass products of star formation. With masses that are below the hydrogen-burning minimum mass (HBMM; $<75 M_{\text {Jup }}$ ), they cannot undergo sustained nucleosynthesis like their higher mass brethren. As such, their compositions stay essentially unchanged from when they formed (e.g., Burrows et al. 1993; Marley et al. 1996). Metal-poor brown dwarfs and very low-mass stars offer unique insights into the young Milky Way as they are objects that formed in the early galaxy and their compositions have not changed much since that time.

Recently, dedicated searches for the old, low-mass population of objects in our galaxy have been carried out using the Wide-field Infrared Survey Explorer (WISE; Kirkpatrick et al. 2014, 2016; Pinfield et al. 2014), UKIDSS Large-Area Survey (ULAS; Zhang et al. 2017b), Two-Micron All Sky Survey (2MASS; Kirkpatrick et al. 2010), and Sloan Digital Sky Survey (SDSS; Sivarani et al. 2009). The totality of the results of these surveys reveals that the lowest-mass stars and brown dwarfs with low metallicity are relatively rare. The very lowest metallicity low-mass stars and brown dwarfs belong to the galactic halo of the Milky Way and are the oldest objects in the galaxy. At a slightly younger age and higher metallicity are the members of the thick-disk. Both populations have high space velocities and are very uncommon in the solar neighborhood. Only $\sim 6 \%$ and $<1 \%$ of the brown dwarfs in the vicinity of the Sun are expected to be part of either the thick-disk or halo populations, respectively (Gazzano et al. 2013). These brown dwarfs have primordial compositions and are excellent testing grounds for models at low metallicities.

$\mathrm{L} / \mathrm{T}$ transition subdwarfs, in particular, are of key interest in studying the effects of metallicity on cloud physics in

\footnotetext{
* This paper includes data gathered with the $6.5 \mathrm{~m}$ Magellan Telescopes located at Las Campanas Observatory, Chile.

${ }^{6}$ NASA Sagan Fellow.
}

atmospheric models. The transition between $\mathrm{L}$ and $\mathrm{T}$ dwarfs, in the case of solar-metallicity brown dwarfs, is a rapid evolution from cloudy to clear atmospheres (e.g., Ackerman \& Marley 2001; Burrows et al. 2001; Burgasser et al. 2002; Marley et al. 2002) and takes place over a narrow effective temperature range of only 200-300 K (e.g., Kirkpatrick 2005). Late-L and early-T subdwarfs, then, are excellent objects to study the role that metallicity plays in the evolution of brown dwarfs and their atmospheres. Due to the nature of the $\mathrm{L} / \mathrm{T}$ transition, however, this spectral range is comparatively devoid of objects, even more so than the late-T subdwarfs, which are not readily detected due to their intrinsically faint near-infrared magnitudes (only four late-T subdwarfs known, discussed in Section 5).

There has also been evidence that there is a "subdwarf gap" that divides stellar and substellar objects at late ages on a colormagnitude diagram (e.g., Kirkpatrick et al. 2016; Zhang et al. 2017a). Stellar theory predicts that at $\sim 10 \mathrm{Gyr}$, the lowest-mass stars and brown dwarfs diverge in their observable properties due to the different energy production process above and below the hydrogen-burning minimum mass (e.g., Chabrier \& Baraffe 1997; Burrows et al. 2001; Burgasser 2004). Lowmass stars and brown dwarfs are more difficult to distinguish at younger ages but evolution models predict the stellar/substellar boundary occurs at higher temperatures for solar-metallicity objects ( L2.5; Dieterich et al. 2014) and brown dwarfs have spectral types as early as M6 at very young ages (e.g., Burrows et al. 2001; Baraffe et al. 2015; Dupuy \& Liu 2017). Searches for low-metallicity objects reveal that the subdwarf gap appears to be between mid- and late-L dwarfs (Kirkpatrick et al. 2016; Zhang et al. 2017a). Late-L/early-T subdwarfs, then, would be on the substellar side of this region. The exact extension of the gap, however, has yet to be determined, and locating the edges is of keen interest in the study of low-metallicity objects.

Given the rarity of halo/thick-disk brown dwarfs, finding even one new object to study is a significant step forward in understanding this population. An even more important 
step to understanding old, low-metallicity objects and their atmospheres is filling in the gap between late- $\mathrm{L}$ and late- $\mathrm{T}$ subdwarfs. As part of the AllWISE2 Motion Survey (hereafter AllWISE2; Kirkpatrick et al. 2016), we have possibly identified the second object in this spectral range that also has kinematics consistent with the population of thick-disk/halo objectsWISE J071121.36-573634.2.

In Section 2, we discuss how we identified this object, and in Section 3, we outline our observations and data reduction. We discuss the spectral classification of WISE J0711-5736 in Section 4 and put it in context of the population of halo/thickdisk objects in Section 5.

\section{Candidate Selection}

The AllWISE1 and AllWISE2 Motion Surveys (Kirkpatrick et al. 2014, 2016) were searches designed to leverage the multiepoch mid-infrared observations of the entire sky and identify objects with proper motions detectable over the 1-year period of the AllWISE mission. Objects with such high apparent motions are either located in the solar neighborhood or have inherently large space velocities. The AllWISE1 Motion Survey imposed an rchi2/rchi2_pm $>1.03$, selecting objects where the $\chi^{2}$ value of the stationary fit was at least $3 \%$ higher than the $\chi^{2}$ of the motion fit. This criteria was removed for the AllWISE2 Motion Survey allowing objects with smaller motions to potentially be recovered in the second iteration of the survey. An interesting subset of the motion sources are ones that do not have counterparts in surveys at shorter wavelengths (e.g., SDSS and 2MASS) - typically late-T and Y dwarfs. The model predictions for the spectral energy distributions of such objects peak at $\sim 4-12 \mu \mathrm{m}\left(700 \mathrm{~K}>T_{\text {eff }}>250 \mathrm{~K}\right)$ so they are very often undetected in optical and near-IR surveys. We, therefore, created a devoted search for similar objects.

We published 58 WISE-only candidates from AllWISE1 in Kirkpatrick et al. (2014). We implemented the same type of selection criterion for WISE-only sources in AllWISE2, selecting objects that did not have counterparts in 2MASS or SDSS. After visual verifications (details in Section 2 of Kirkpatrick et al. 2016), we ended up with 11 WISE-only objects from AllWISE2, for a total of 69 candidate late-T and Y dwarfs from both AllWISE1 and AllWISE2. The WISE magnitudes and proper motions of the 11 new WISE-only AllWISE2 candidates are presented in Table 1. One AllWISE1 object turned out to be an important new discovery: the Y dwarf WISEA J085510.74-071442.5 (Luhman 2014). Four of the candidates from AllWISE2 were also already known $\mathrm{T}$ and $\mathrm{Y}$ dwarfs: WISE J223617.60+510551.8 (T5; Mace et al. 2013a), WISE J104752.35+212417.2 (T6.5; Burgasser et al. 1999), WISE J115013.85+630241.5 (T8; Kirkpatrick et al. 2011), and WISE J140518.39+553421.3 (Y0pec?; Kirkpatrick et al. 2011). Two of the known ultra-cool dwarfs-WISE J104752.35+212417.2 and WISE J223617.60 +510551.8 - actually did have 2MASS counterparts, but their motions are high enough that they were not recognized in the 2MASS images. For the rest of the analysis, we only consider the 64 new candidate ultra-cool dwarfs.

All of the new objects had motions that were quite small and at the limit of bye-eye detection. As the longest baseline for any of the objects was only 0.5 year, we turned to the new data from the NEOWISE Reactivation mission (Mainzer et al. 2011) to provide a longer baseline. We used the WISE co-add tool ${ }^{7}$

\footnotetext{
http://irsa.ipac.caltech.edu/applications/ICORE/
}

developed at IPAC to combine images for each of the 64 new candidates for comparison. We created two co-added images: one from the observations during the first observational epoch (early 2010; WISE All-Sky) and one from observations during the last epoch (late 2015; NEOWISE-R). This gave a baseline of $>5$ years for most candidates, sufficient to authenticate their motion. We determined the motions by measuring the centroids of the objects in each co-added image with DAOFIND in IRAF and calculating a linear fit between the objects at each of the two epochs. Because the epochs are $>5$ years apart for most candidates, the space motion of the objects dominates the observed motion and the parallax is a small, second-order component. Calculated proper motions based on the longer baseline co-added images are presented in Table 1. One object, WISE J082811.56-443738.1, turned out to increase in brightness rather than have any motion so we did not follow this object up.

Only one new candidate showed clear motion-WISE J071121.36-573634.2 (hereafter WISE J0711-5736). The calculated motions of all of the other new candidates were much smaller than the motions from AllWISE and are likely not brown dwarfs in the solar neighborhood. The co-added $W 2$ images of WISE J0711-5736 from the first and last observational epoch are shown in Figure 1. From these images, we calculated a more accurate proper motion of WISE J0711-5736 (Table 2) compared to the AllWISE proper motion estimates (Table 1).

This object, however, does not have the characteristic infrared colors of a late-T or Y dwarf. Instead, the colors appeared to be that of a late-L or early-T dwarf based on the $W 1-W 2$ versus spectral type relation from Kirkpatrick et al. (2011). With this assumption and an estimated absolute $W 2$ magnitude for this spectral range from Faherty et al. (2016; Figure 3), we calculated a photometric distance $(36-39 \mathrm{pc})$ and tangential velocity $\left(165-200 \mathrm{~km} \mathrm{~s}^{-1}\right)$ consistent with the population of galactic halo/thick-disk objects. We also calculated the reduced proper motion $\left(H_{W 2}=W 2+5 \log (\mu)+5\right)$ of this object to be $\approx 19.6 \mathrm{mag}$. From Figure 11 of Pinfield et al. (2014), we see that the $H_{W 2}$ and $W 1-W 2$ values put this object far outside the $100 \mathrm{~km} \mathrm{~s}^{-1}$ curve- - also consistent with being part of at least the thick-disk population. For verification of the spectral type of WISE J0711-5736 we obtained near-IR spectra. The details are outlined in the next section.

\section{Spectroscopic Observations and Data Reduction}

We observed WISE J0711-5736 on UT 2016 December 14 with the Folded-port InfraRed Echellette (FIRE; Simcoe et al. 2008; Simcoe et al. 2013) at the Magellan/Baade telescope in the high-throughput prism mode. The weather was clear with a seeing of $\sim 0$ " 3 . We used the 0 ". 6 slit, yielding a resolving power of $R \sim 450$ in the $0.8-2.45 \mu \mathrm{m}$ wavelength range. We obtained four $120 \mathrm{~s}$ exposures in a nodding ABBA pattern along the slit at airmasses of 1.262-1.275, yielding a signal-to-noise ratio of $\sim 50$ per pixel in the $1.5-1.8 \mu \mathrm{m}$ range. The telluric exposures that were obtained immediately before WISE J0711-5736 were saturated and therefore not usable. The A0-type star HD 35265 observed on UT 2016 January 22 with six $1 \mathrm{~s}$ exposures under clear weather conditions, a seeing of $\sim 0$." 6 , and an airmass of 1.210-1.231, was therefore used to perform the telluric correction. We obtained eleven $1 \mathrm{~s}$ exposures of $\mathrm{NeAr}$ calibration lamps at the beginning of the respective nights to perform the wavelength calibration, as well 
Table 1

AllWISE2 Motion Candidates Lacking 2MASS Counterparts

\begin{tabular}{|c|c|c|c|c|c|c|c|c|}
\hline Designation & $\begin{array}{c}\text { W1 } \\
(\mathrm{mag})\end{array}$ & $\begin{array}{c}W 2 \\
(\mathrm{mag})\end{array}$ & 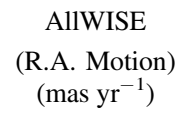 & $\begin{array}{c}\text { AllWISE } \\
\text { (Decl. Motion) } \\
\left(\operatorname{mas~yr}^{-1}\right)\end{array}$ & $\begin{array}{l}\text { Calculated } \\
\mu_{\alpha} \cos (\delta) \\
\left(\text { mas } \mathrm{yr}^{-1}\right)\end{array}$ & $\begin{array}{c}\text { Calculated } \\
\mu_{\delta} \\
\left(\mathrm{mas} \mathrm{yr}^{-1}\right)\end{array}$ & $\begin{array}{l}\text { Spectral } \\
\text { Type }\end{array}$ & $\begin{array}{l}\text { Discovery } \\
\text { Publication }\end{array}$ \\
\hline WISE J003428.12+393153.7 & $15.910 \pm 0.046$ & $14.666 \pm 0.050$ & $663 \pm 224$ & $-1033 \pm 233$ & $-3 \pm 25$ & $-48 \pm 80$ & & 1 \\
\hline WISE J071121.36-573634.2 & $15.092 \pm 0.029$ & $14.627 \pm 0.038$ & $507 \pm 137$ & $998 \pm 145$ & $18 \pm 10$ & $990 \pm 90$ & & 1 \\
\hline WISE J081031.30-475602.8 & $15.741 \pm 0.039$ & $14.398 \pm 0.041$ & $-1215 \pm 272$ & $-902 \pm 274$ & $6 \pm 25$ & $63 \pm 80$ & & 1 \\
\hline WISE J082811.56-443738.1 ${ }^{\mathrm{a}}$ & $14.751 \pm 0.031$ & $12.650 \pm 0.024$ & $512 \pm 83$ & $643 \pm 88$ & $2 \pm 28$ & $18 \pm 85$ & & 1 \\
\hline WISE J102055.17+530859.4 & $15.666 \pm 0.042$ & $14.526 \pm 0.047$ & $1247 \pm 304$ & $1287 \pm 326$ & $-2 \pm 25$ & $31 \pm 80$ & & 1 \\
\hline WISE J104752.35+212417.2 & $15.377 \pm 0.036$ & $13.004 \pm 0.030$ & $-908 \pm 171$ & $-682 \pm 186$ & $-112 \pm 25$ & $-516 \pm 80$ & T6.5 & 2 \\
\hline WISE J115013.85+630241.5 & $16.958 \pm 0.089$ & $13.405 \pm 0.028$ & $330 \pm 198$ & $-1194 \pm 202$ & $63 \pm 25$ & $-540 \pm 80$ & $\mathrm{~T} 8$ & 3 \\
\hline WISE J122738.12-232819.6 & $16.102 \pm 0.058$ & $14.692 \pm 0.061$ & $1897 \pm 317$ & $221 \pm 352$ & $7 \pm 27$ & $33 \pm 85$ & & 1 \\
\hline WISE J140518.39+553421.3 & $18.765 \pm 0.396$ & $14.097 \pm 0.037$ & $-1862 \pm 326$ & $-324 \pm 334$ & $-265 \pm 25$ & $187 \pm 80$ & Y0(pec?) & 3 \\
\hline WISE J153747.73+181151.3 & $14.198 \pm 0.025$ & $12.220 \pm 0.022$ & $-228 \pm 64$ & $-367 \pm 69$ & $5 \pm 25$ & $48 \pm 80$ & & 1 \\
\hline WISE J223617.60+510551.8 & $13.827 \pm 0.025$ & $12.499 \pm 0.025$ & $573 \pm 99$ & $-118 \pm 89$ & $70 \pm 27$ & $358 \pm 85$ & T5 & 4 \\
\hline
\end{tabular}

Note. Discovery papers are: (1) This work, (2) Burgasser et al. (1999), (3) Kirkpatrick et al. (2011), (4) Mace et al. (2013a).

${ }^{a}$ WISE J082811.56-443738.1 is a flux transient rather than a motion object. 

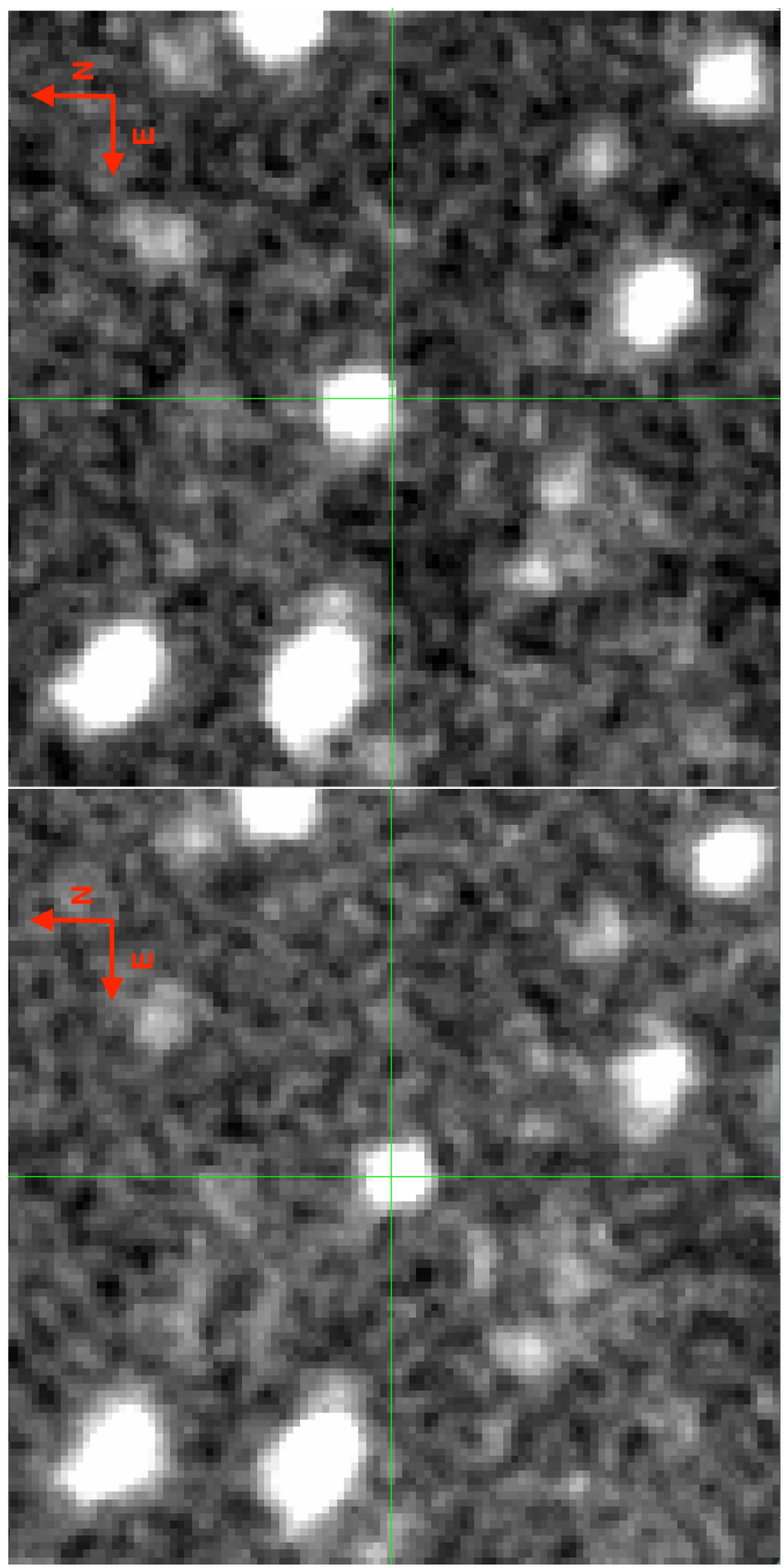

Figure 1. Co-added $2^{\prime} \times 2^{\prime}$ images from WISE in W2. The first image is from UT 2010 May 4 (WISE All-Sky), the second is from UT 2015 November 16 (NEOWISE-R). 
Table 2

Object Properties

\begin{tabular}{lc}
\hline \hline Identifier & WISE J071121.36-573634.2 \\
R.A. & $07: 11: 21.36$ \\
Decl. & $-57: 36: 34.20$ \\
$W 1$ & $15.092 \pm 0.029 \mathrm{mag}$ \\
$W 2$ & $14.627 \pm 0.038 \mathrm{mag}$ \\
$W 3$ & $12.504 \pm 0.500 \mathrm{mag}$ \\
$W 4$ & $9.558 \pm 0.500 \mathrm{mag}^{-1}$ \\
Calculated $\mu_{\alpha} \cos (\delta)^{\mathrm{a}}$ & $18 \pm 10 \mathrm{mas} \mathrm{yr}^{-1}$ \\
Calculated $\mu_{\delta}{ }^{\mathrm{a}}$ & $990 \pm 90 \mathrm{mas} \mathrm{yr}^{-1}$ \\
$H_{W 2}$ & $19.61 \pm 0.02 \mathrm{mag}$ \\
\hline
\end{tabular}

Note.

${ }^{\text {a }}$ Calculated motions are based on the positions on UT 2010 May 4 (WISE AllSky) and 2015 November 16 (NEOWISE-R).

Table 3

Inferred Properties of WISE J0711-5736

\begin{tabular}{lc}
\hline \hline Spectral Type & sd T0 \pm 1 \\
\hline From Faherty et al. (2016) & \\
\hline$M_{W 2}$ & $11.8 \pm 0.5 \mathrm{mag}$ \\
Photometric Distance & $36.8 \pm 4.2 \mathrm{pc}$ \\
Tangential Velocity & $173 \pm 22 \mathrm{~km} \mathrm{~s}^{-1}$ \\
\hline From E. Gonzales et al. (2018, in preparation) & \\
\hline$M_{W 2}$ & $12.3 \pm 0.5 \mathrm{mag}$ \\
Photometric Distance & $28.9 \pm 4.2 \mathrm{pc}$ \\
Tangential Velocity & $149 \pm 22 \mathrm{~km} \mathrm{~s}^{-1}$ \\
\hline
\end{tabular}

Note. Uncertainties represent the values for the range of possible spectral types -L9-T1.

as eleven exposures of low- and high-voltage dome flat fields to correct for pixel response variation.

We reduced the data with the Interactive Data Language (IDL) Firehose v2.0 package (Bochanski et al. 2009; Gagné et al. 2015\%; see Gagné et al. 2015 for more details on the reduction package). We extracted the spectra using an optimal extraction approach. The local background was modeled using a basis spline fit to the masked aperture profile and subtracted it from the spectra. We subsequently extracted the spectra using a weighted profile extraction approach. We wavelength-calibrated and mediancombined the resulting spectra using a modified version of the Spextool routine xcombspec (Cushing et al. 2004). Finally, we corrected for telluric absorption and flux-calibrated the spectra with xtellcor_general.pro using the A0 calibration star.

As our telluric standard was observed 11 months prior to WISE J0711-5736 and the precipitable water content in the atmosphere was likely not the same on the two nights, we performed the same type of telluric correction using two additional standards. Observed at an airmass of $\sim 1.07$ on 2016 January 22, HD 102338 was located in a region of sky with lower water content than HD 35265 (airmass $\approx 1.22$ ) and HD 149818, observed at an airmass of $\sim 1.83$ on 2016 January 22, was located in a region of sky with higher water content. Figure 2 shows the comparison of WISE J0711-5736 corrected with all three telluric standards and smoothed for clarity. As we can see, the depths of all of the water bands $(1.10-1.18 \mu \mathrm{m}$,

\footnotetext{
8 Avaliable at https://github.com/jgagneastro/FireHose_v2/tree/v2.0.
}

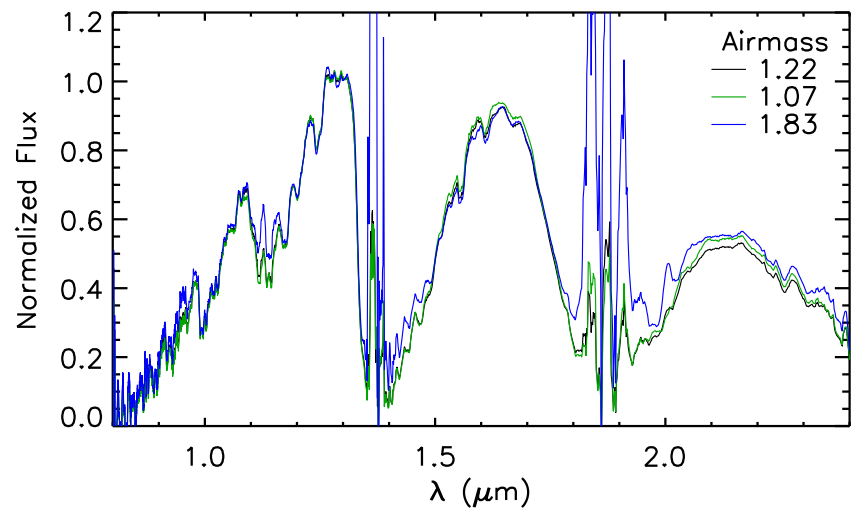

Figure 2. Comparison of smoothed FIRE prism spectra of WISE J0711-5736 telluric corrected with HD 35265 (airmass $\approx 1.22$; black), HD 102338 (airmass $\approx 1.07$; green) and HD 149818 (airmass $\approx 1.83$; blue). Spectra are normalized to the average flux in the $1.25-1.30 \mu \mathrm{m}$ region.

$1.30-1.45 \mu \mathrm{m}$, and $1.70-1.95 \mu \mathrm{m})$ are similar between the spectra corrected with two standards at low and moderate airmasses (HD 102338 and HD 35265). The spectrum that was corrected with the standard at a higher airmass (HD 149818) has less water absorption. However, the residual telluric features at $2.0-2.1 \mu \mathrm{m}$ indicate that the spectrum corrected with HD 149818 was over-corrected and too much water absorption was removed. We also note that the (sub)stellar water bands are wider than the telluric ones due to atmospheric pressures, so the wings are not affected by the telluric correction, as Figure 2 shows.

From this analysis, we confirm that performing a correction with HD 35265 was sufficient to remove all of the telluric absorption. Thus, we conclude that the depths of the water bands are intrinsic to WISE J0711-5736 and are not a consequence of correcting with a telluric standard that was observed on a night with different precipitable water content.

\section{Spectral Classification and Kinematics}

In order to classify WISE J0711-5736, we compare our FIRE spectrum to spectra of published objects and to theoretical spectra. When comparing to published spectra, we smooth our medium-resolution spectrum to the resolution of the comparison object using a least-squares quadratic interpolation. All spectra are normalized to the median flux value in the $1.25-1.30 \mu \mathrm{m}$ region.

\subsection{Late-L/Early-T Subdwarf}

In Figure 3, we compare our FIRE spectrum of WISE J0711-5736 to the closest matching spectra of each type of L9-T1 dwarf from the SpeX Prism Library. From this comparison, we see that WISE J0711-5736 has more FeH and $\mathrm{K} \mathrm{I}$ absorption than all of the comparison objects and very little CO absorption at $\sim 2.3 \mu \mathrm{m}$. We also see that WISE J0711-5736 has deeper water bands.

All of these features are characteristic of an old, metal-poor ultra-cool dwarf (Burgasser et al. 2008b). Low-metallicity brown dwarfs typically have increased $\mathrm{FeH}$ absorption and weak signatures of CO. These metal-poor objects are also old with high surface gravities, which leads to greater line strengths of the pressure-sensitive alkali species, namely KI. As Burgasser et al. (2008b) details; however, these characteristics cannot explain all of the features in the spectra of subdwarfs, 


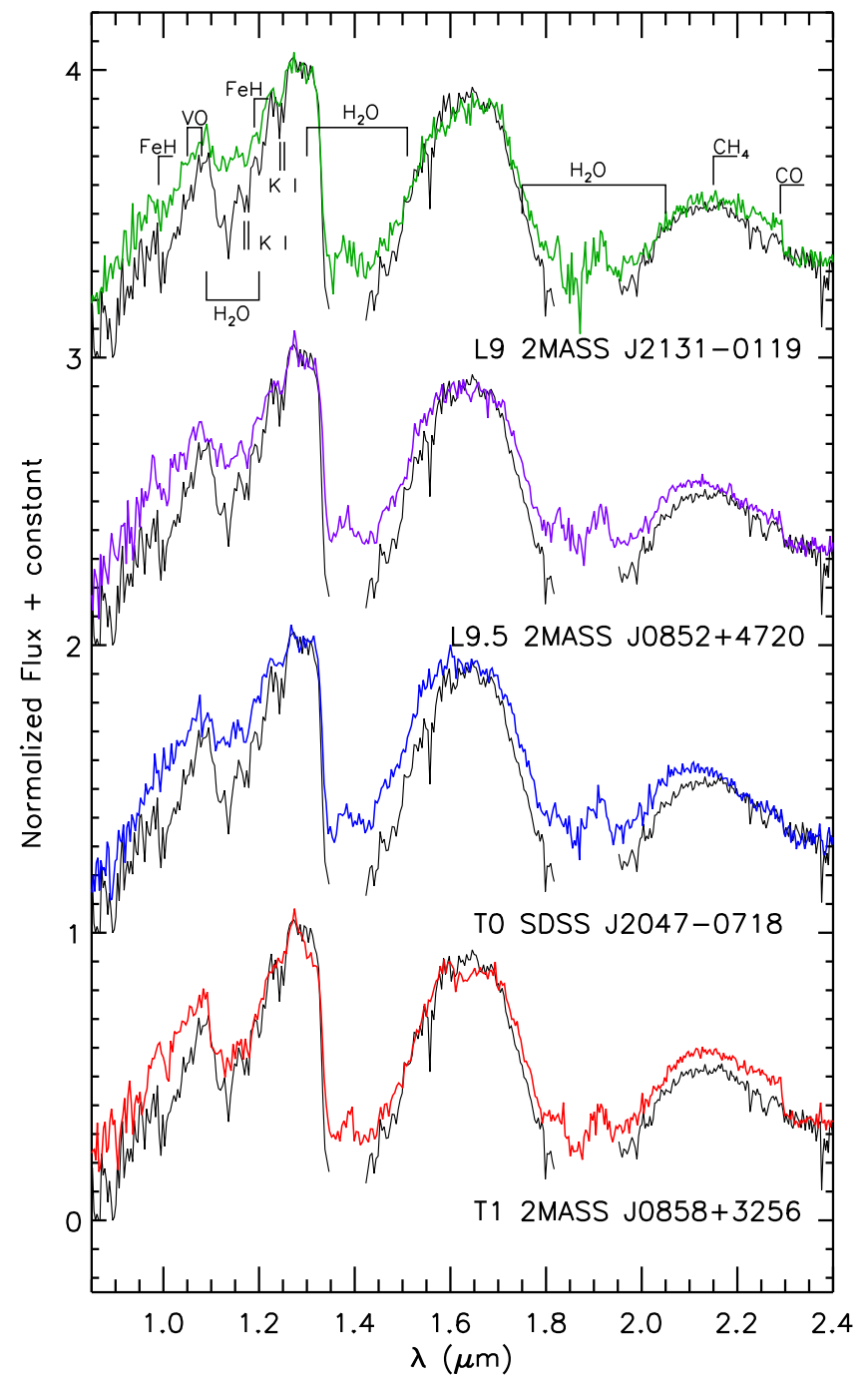

Figure 3. Our FIRE spectrum of WISE J0711-5736 (black) compared to SpeX Prism Library spectra of L9-T1 dwarfs. The FIRE spectrum has been smoothed to the resolution of the SpeX spectra. Comparison spectra are: L9 (2MASS J21315444-0119374; Chiu et al. 2006); L9.5 (2MASS J08523490+4720359; Burgasser et al. 2010); T0 (SDSS J204749.61-071818.3; Burgasser et al. 2010); T1 (2MASS J08583467+3256275; Burgasser et al. 2010). Spectra are normalized to the average flux in the $1.25-1.30 \mu \mathrm{m}$ region.

particularly the strong $\mathrm{H}_{2} \mathrm{O}$ bands. For that, thin and/or largegrain condensate clouds are needed to reduce the contrast between the $J$-band peak and $1.4 \mu \mathrm{m}$ water band.

Although these are typical characteristics of low-metallicity L dwarfs, we assume they are also the features of late-L/early$\mathrm{T}$ subdwarfs. One defining characteristic of subdwarfs that we cannot match is the blue $J-K$ color-our NIR spectrum does not show enhanced collision-induced $\mathrm{H}_{2}$ absorption $\left(\mathrm{CIA} \mathrm{H}_{2}\right)$ in the $H$ - and $K$-bands. If WISE J0711-5736 had enhanced CIA $\mathrm{H}_{2}$ absorption, the $H$ - and $K$-bands would also appear flatter as is seen in other subdwarfs (Figure 4). As this is not the case, we conclude that this object truly does not have a blue $J-K$ color, and the redder $J-K$ slope is not a consequence of instrumental effects or an improperly calibrated spectrum. The derived color can be checked via future follow-up either by obtaining another higher signal-to-noise spectrum or by obtaining $J$ and $K$ photometry.

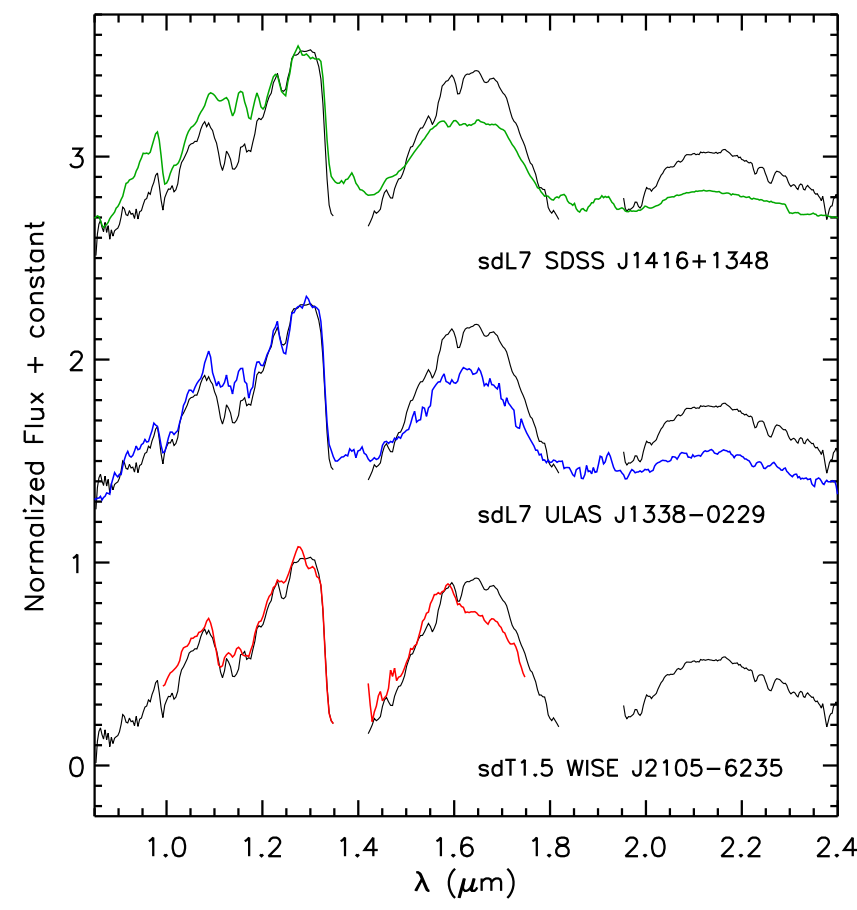

Figure 4. Our FIRE spectrum of WISE J0711-5736 (black) compared to the SpeX Prism spectrum of SDSS J141624.08+134826.7 (sdL7; Schmidt et al. 2010), the FIRE Prism spectrum of ULAS J133836.97-022910.7 (sdL7; Zhang et al. 2017b), and the Gemini Flamingos-2 spectrum of WISE J210529.08-623558.7 (sdT1.5; Luhman \& Sheppard 2014). All spectra have been smoothed to the resolution of the SpeX spectrum for a more direct comparison. Spectra are normalized to the average flux in the $1.25-1.30 \mu \mathrm{m}$ region.

We have also compared our spectrum of WISE J0711-5736 to two L7 subdwarfs (SDSS J141624.08+134826.7, Schmidt et al. 2010; ULAS J133836.97-022910.7, Zhang et al. 2017b) and a suspected T1.5 subdwarf (WISE J210529.08-623558.7, Luhman \& Sheppard 2014) in Figure 4. These are a few of the only late-L/early-T subdwarfs known to date. From Figure 4, we see that the strengths of the $\mathrm{KI}$ and $\mathrm{FeH}$ features of WISE J0711-5736 match those of the sdL7 dwarf ULAS J1338-0229 as well as the lack of CO absorption. Both SDSS J1416+1348 and ULAS J1338-0229 have bluer $J-K$ colors and shallower $\mathrm{H}_{2} \mathrm{O}$ bands, but this could be a consequence of the different spectral types. The depths of the $\mathrm{H}_{2} \mathrm{O}$ bands of WISE J0711-5736 are more comparable to those of WISE J2105-6235 but the $\mathrm{KI}$ and $\mathrm{FeH}$ features are stronger. The long-wavelength side of the $H$-band appears to be suppressed by CIA $\mathrm{H}_{2}$, but the effects on the $K$-band are unknown because the F2 spectrum of WISE J2105-6235 only covers the $J$ - and $H$-bands.

Although WISE J0711-5736 lacks the characteristic blue NIR color of a typical low-metallicity object, all other signs point to it being part of the old galactic population. Thus, we tentatively conclude that this is a T0 \pm 1 subdwarf.

We have also compared our spectrum to theoretical spectra. The set of models that most comprehensively cover the temperature, gravity, and metallicity parameters are the spectra from Burrows et al. (2006). We have compared our FIRE spectrum of WISE J0711-5736 to model spectra that have $T_{\text {eff }}=1300-1500 \mathrm{~K}$, $\log g=4.5-5.5$ and metallicities between 0.3 and 3 times solar (Figure 5). We can see from Figure 5 that when the models are compared to each other, the low-metallicity models have deeper 


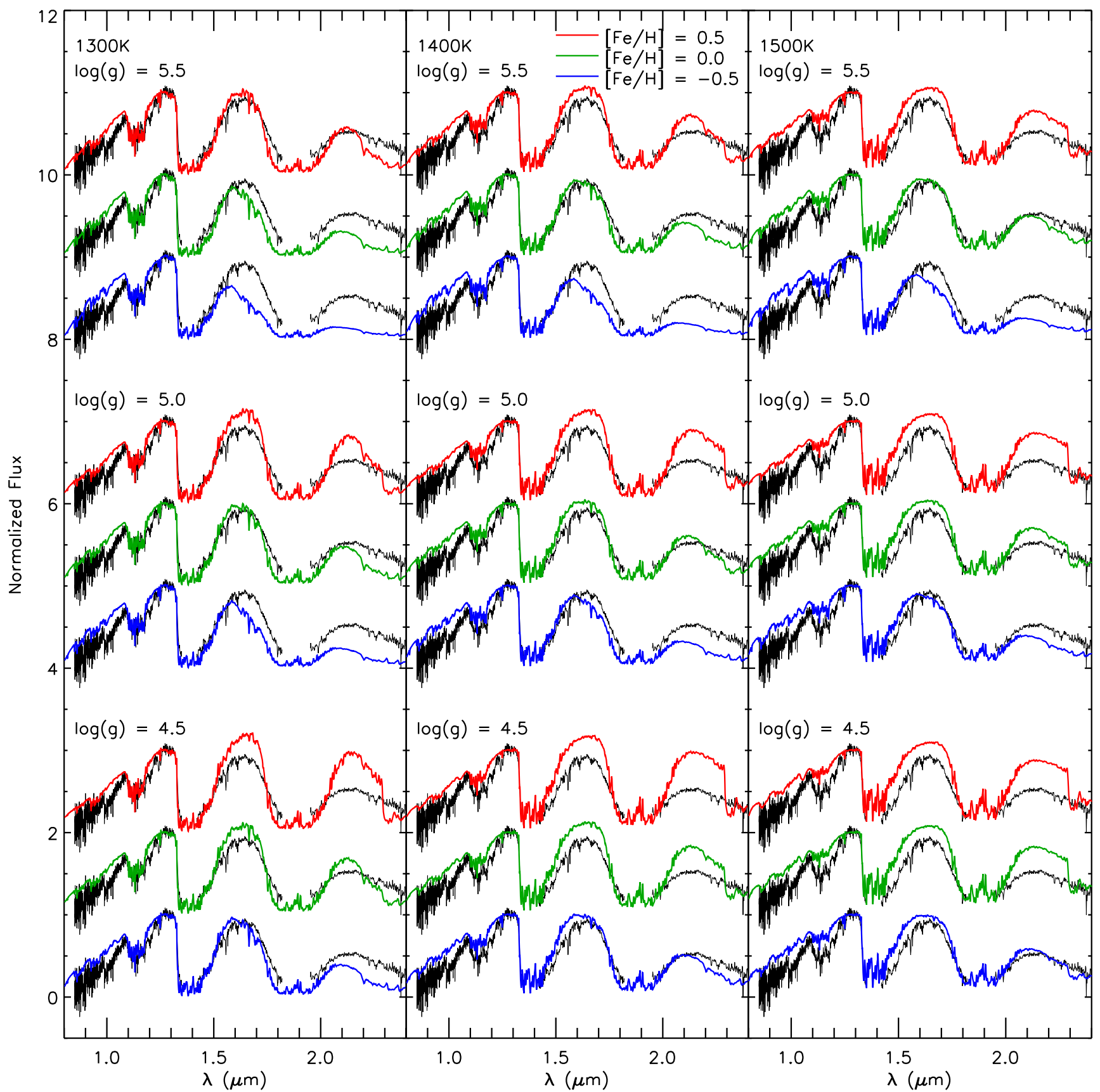

Figure 5. The FIRE spectrum of WISE J0711-5736 (black) compared to theoretical spectra from Burrows et al. (2006). The low-metallicity models (blue) and lowgravity models (lowest sets) seem to fit the spectrum of WISE J0711-5736 best; however, the fits are quite poor. Spectra are normalized to the average flux in the $1.25-1.30 \mu \mathrm{m}$ region.

$\mathrm{FeH}$ and $\mathrm{K}$ I features and a lack of $\mathrm{CO}$ absorption compared to the higher metallicity models. As this is similar to what we observe when we compare WISE J0711-5736 to other L and T dwarfs, we reason that this object likely also has a low metallicity, despite the poor model fits and apparent lack of enhanced CIA $\mathrm{H}_{2}$ absorption. The lower surface gravity models appear to provide better comparisons visually, but the fits are still quite poor. The fact that the best fits appear to have both a low surface gravity and a low metallicity is puzzling, as we would expect a low-metallicity object to be older, and hence, have higher surface gravity.

The overall best-fitting model is the $1500 \mathrm{~K}, \log g=5.5$ and $0.3 \times$ solar-metallicity model (which we will denote as $15-5.5$ $0.3 \mathrm{X}$ ) with a reduced $\chi^{2}$ of 0.19 . The best-fitting models in the
$J$-, $H$-, and $K$ - bands are the 15-5.5-0.3X (reduced $\chi^{2}=0.17$ ), 15-4.5-0.3X (reduced $\chi^{2}=0.16$ ), and 14-4.5-3X (reduced $\left.\chi^{2}=0.42\right)$, respectively. The inconsistency between the bestfit models and the poor visual similarity of these best-fit models with the spectrum of WISE J0711-5736 does not allow us to make any definitive conclusions about the nature of this object.

\subsection{Unresolved Multiple?}

We also compared the spectrum of WISE J0711-5736 to objects that were classified as peculiar in the SpeX Prism Library and to templates of spectral binaries. We created our templates by normalizing all single $\mathrm{L}$ and $\mathrm{T}$ dwarfs in the SpeX 


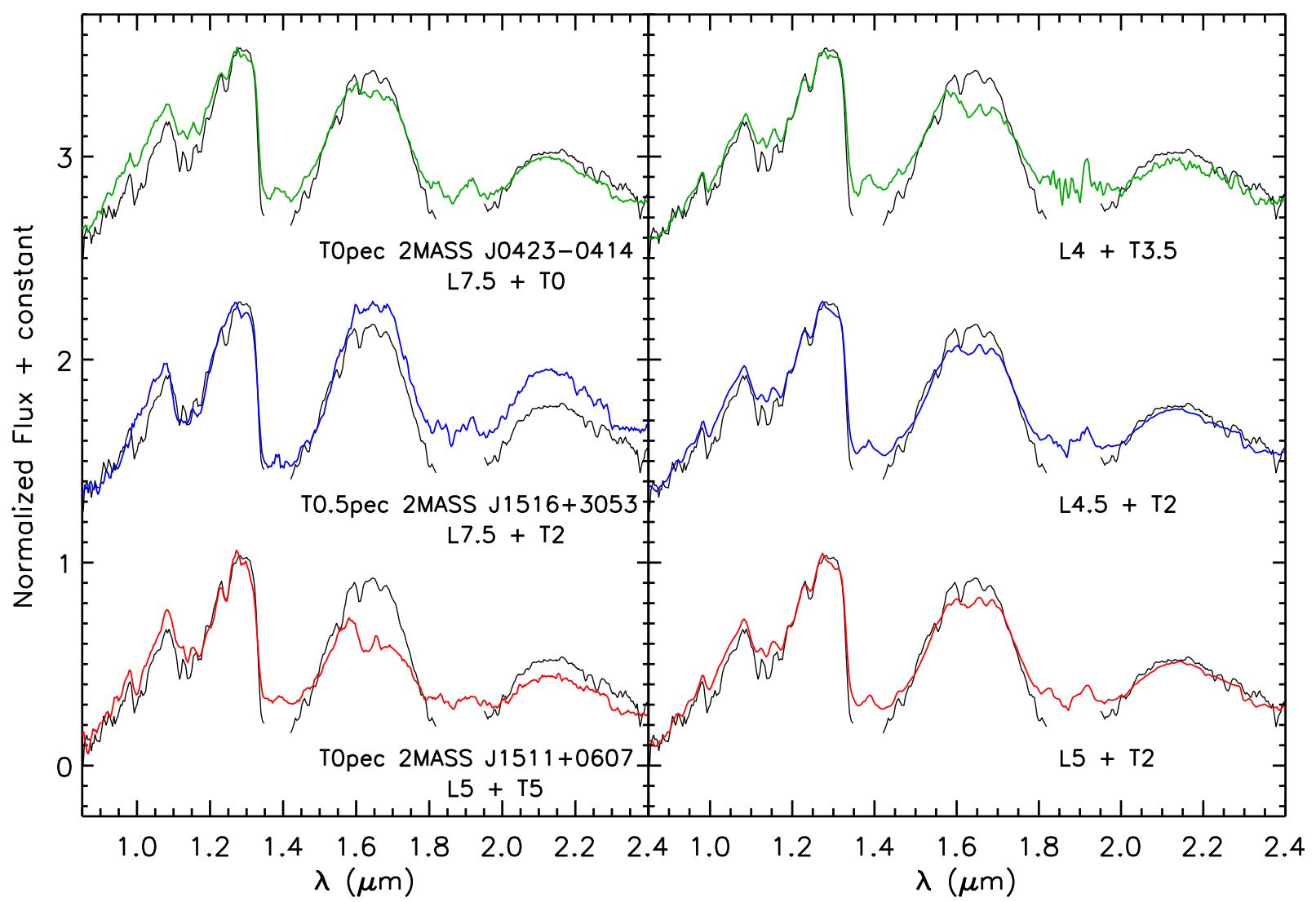

Figure 6. Our FIRE spectrum of WISE J0711-5736 (black) compared to SpeX Prism peculiar spectra (left) and spectral binary templates (right). The FIRE spectrum has been smoothed to the resolution of the SpeX spectra. Comparison spectra for the peculiar objects are: T0pec (2MASS J04234858-0414035; Burgasser et al. 2004); T0.5pec (SDSS J151642.97+305344.5; Burgasser et al. 2010); T0pec (2MASS J15111466+0607431; Chiu et al. 2006). The spectra used in the spectral binary templates are: L4 (2MASS J04070752+1546456; Burgasser et al. 2008a) and T3.5 (SDSS J153417.05+161546.1; Chiu et al. 2006); L4.5 (2MASSI J0652307 +471034; Burgasser et al. 2010) and T2 (SDSS J024749.90-163112.6; Chiu et al. 2006); L5 (2MASSW J1239272+551537; Burgasser et al. 2010) and T2 (SDSS J024749.90-163112.6; Chiu et al. 2006). Spectra are normalized to the average flux in the $1.25-1.30 \mu \mathrm{m}$ region.

Prism Library to the flux in the $1.25-1.30 \mu \mathrm{m}$ region, scaling them to their spectral-type dependent absolute magnitudes given in Filippazzo et al. (2015), and summing the pairs of resulting spectra. The best matches are shown in Figure 6; however, none of the spectra can reproduce all of the features in the spectrum of WISE J0711-5736, in particular the slopes in the $H$-band. Interestingly, the best matching peculiar spectra in the $J$-band are 2MASS J04234858-0414035 (Burgasser et al. 2004), 2MASS J15111466+0607431 (Chiu et al. 2006), and SDSS J151642.97+305344.5 (Burgasser et al. 2010), all of which are confirmed or suspected binaries (Burgasser et al. 2006, 2010; Bardalez Gagliuffi et al. 2015). The L7.5 + T0 spectrum of 2M J0423-0414 fits the $H$ - and $K$-bands quite well but matches the $J$-band only marginally better than the single spectra of Figure 3. The L7.5 + T2 spectrum of SDSS J1516+3053 matches the depth of the water absorption bands but fails to match the FeH and K I absorption features and the overall slope of WISE J0711-5736. The L5 + T5 spectrum of $2 \mathrm{M} \mathrm{J} 1511+0607$ fits the $J$-band of WISE J0711-5736 extremely well but fails to match the $H$ - and $K$ bands, mostly due to the contribution of the T5 in $2 \mathrm{M}$ $\mathrm{J} 1511+0607$.

From these comparisons, we see that as the fit in the $J$-band improves with combinations of earlier $\mathrm{L}$ dwarfs and later $\mathrm{T}$ dwarfs, the fit in the $H$ - and $K$-bands becomes worse. This, along with the apparent lack of $\mathrm{CH}_{4}$ absorption disfavors the possibility that this object is a spectral binary. The redder $J-K$ slope of WISE J0711-5736 compared to subdwarfs and lack of CIA by $\mathrm{H}_{2}$, however, potentially supports the binary explanation.

The best fits from the spectral binary template fitting seem to produce better matches (right panel of Figure 6); however, the templates still cannot match the strength of the $\mathrm{KI}$ and $\mathrm{FeH}$ features. Quantitative measurements show these templates provide no, or no significant, improvement over the fit to the sdT1.5 dwarf WISE 2105-6235 (reduced $\chi^{2}=0.30,0.17$ and 0.17 , respectively, versus 0.19 for WISE 2105-6235). We also note that at least one spectrum in each of the templates is itself a suspected binary or other peculiar object: the T3.5 dwarf SDSS J153417.05+161546.1 (Chiu et al. 2006) is a T1.5 +T5.5 binary (Liu et al. 2006); the L4.5 dwarf 2MASSI J0652307+471034 (Burgasser et al. 2010) is potentially young (Cruz et al. 2007); the T2 dwarf SDSS J024749.90-163112.6 (Chiu et al. 2006) is a candidate T0+T7 binary (Burgasser et al. 2010); and the L5 dwarf 2MASSW J1239272+551537 (Burgasser et al. 2010) is an L5+L6 binary (Radigan et al. 2013). Because one spectrum in each template is itself a suspected binary, the spectra are in fact triple templates. As the scenario of a young or thin-disk triple system moving with such large proper motion is unlikely, we also tentatively rule 
out this possibility. However, we cannot altogether dismiss the possibility of unresolved multiplicity.

\subsection{Kinematics}

Using the absolute magnitude versus spectral type polynomial from Faherty et al. (2016) for a T0 \pm 1 dwarf, we estimate that WISE J0711-5736 has an absolute W2 magnitude of $\sim 11.8 \mathrm{mag}$, giving a photometric distance of $37 \mathrm{pc}$. The proper motion of this object at the photometric distance corresponds to a tangential velocity of $\sim 170 \mathrm{~km} \mathrm{~s}^{-1}$. The relations of Faherty et al. (2016), however, were calibrated using normal field objects, and we expect the absolute $W 2$ magnitudes of late-L/early-T subdwarfs to be slightly fainter than those of normal objects of the same spectral type as that is the behavior seen in higher mass brown dwarfs and lowmass stars. This would mean our distance and velocity values are slightly overestimated. Using the absolute magnitude versus spectral type relations from Gonzales et al. (2017, in preparation) for subdwarfs up to L5 and doing a naive extension to T0 spectral types, WISE J0711-5736 should have an absolute magnitude of $\sim 12.3 \mathrm{mag}$, giving a photometric distance of $29 \mathrm{pc}$. The proper motion then corresponds to $\sim 150 \mathrm{~km} \mathrm{~s}^{-1}$; a much smaller value.

WISE has an angular resolution of 6". 1 in $W 1$ and 6!" 4 in $W 2$. If WISE J0711-5736 is a multiple object, the components would have a separation of less than $226 \pm 26 \mathrm{au}$ or $177 \pm 26 \mathrm{au}$, using the two distance estimates $37 \mathrm{pc}$ and $29 \mathrm{pc}$, respectively, to be unresolved with WISE.

\section{Halo/Thick-disk Brown Dwarfs}

From studies of stars, tangential velocities of objects in the thick-disk have been found to be $\sim 85-180 \mathrm{~km} \mathrm{~s}^{-1}$ (e.g., Fuhrmann 1998; Feltzing et al. 2003; Soubiran et al. 2003) and $\sim 200-300 \mathrm{~km} \mathrm{~s}^{-1}$ in the halo (e.g., Chiba \& Beers 2000; Schilbach et al. 2009; Mace et al. 2013b). With a tangential velocity of $\sim 170 \mathrm{~km} \mathrm{~s}^{-1}$, WISE J0711-5736 lies right on the cusp between these populations. Based on the membership probability distributions calculated by Dupuy \& Liu (2012), WISE J0711-5736 has a $>90 \%$ probability of being part of the thick-disk and $<10 \%$ probability of being a member of either the halo or thin-disk populations. Using the tangential velocity calculated that uses the relation from Gonzales et al. (2017, in preparation), WISE J0711-5736 has a membership probability of $50 \%-90 \%$ for the thick-disk, $\leqslant 10 \%$ for the thin-disk, and $<10 \%$ for the halo. If WISE J0711-5736 were an unresolved binary, it would be a factor of $\sim 30 \%-40 \%$ further away, and so would have a proportionately higher tangential velocity: putting it further into the thick-disk kinematics.

The comparison of WISE J0711-5736 with solar-metallicity objects and theoretical spectra with $[\mathrm{Fe} / \mathrm{H}]$ values between 0.0 and -0.5 indicates a low metallicity, making it consistent with either the thick-disk or halo populations (Reddy et al. 2006; Lépine et al. 2007; Zhang et al. 2017a). However, this is only tentative as the model fits are quite poor.

Only a handful of $\mathrm{T}$ dwarfs have been confirmed to be a part of the thick-disk or halo populations, most of which are late-T dwarfs-WISE J200520.38+542433.9 (T8; $v_{\tan } \approx 110 \mathrm{~km} \mathrm{~s}^{-1}$;

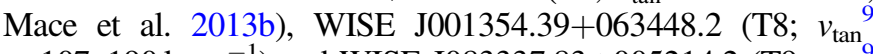
$\approx 107-190 \mathrm{~km} \mathrm{~s}^{-1}$ ) and WISE J083337.83+005214.2 (T9; $v_{\tan }{ }^{9}$

\footnotetext{
9 Assuming a photometric distance using mid-infrared magnitudes, as we do in this work.
}

$\approx 126-231 \mathrm{~km} \mathrm{~s}^{-1}$; Pinfield et al. 2014), and ULAS J131610.28 +075553.0 (T6.5; $v_{\tan } \approx 240-340 \mathrm{~km} \mathrm{~s}^{-1}$; Burningham et al. 2014). The latest L-type brown dwarfs that are part of the thickdisk or halo populations are L7 dwarfs (e.g., Burgasser et al. 2003; Zhang et al. 2017b). There has, so far, been a lack of discoveries of low-metallicity late-L and early-T brown dwarfs. Only one early-T dwarf, WISE J210529.08-623558.7 (T1.5; $v_{\tan }=176 \pm 25 \mathrm{~km} \mathrm{~s}^{-1}$; Luhman \& Sheppard 2014), has been found to potentially have kinematics and a metallicity consistent with these populations. The discovery of WISE J0711-5736 potentially brings the number of objects in this observational gap up to two.

More detailed metallicity measurements and kinematics are needed to confidently determine to which population this object belongs: halo or thick-disk.

\section{Conclusions}

We have determined that our candidate object, WISE J071121.36-573634.2, is potentially an sd T0 \pm 1 dwarf. The estimated absolute $W 2$ magnitude of this object puts it at $\sim 37 \mathrm{pc}$ with a tangential velocity of $\sim 170 \mathrm{~km} \mathrm{~s}^{-1}$, or $\sim 29 \mathrm{pc}$ with a tangential velocity of $\sim 150 \mathrm{~km} \mathrm{~s}^{-1}$. The properties of WISE J0711-5736 are generally consistent with those of the thick-disk population. Using either of the distance estimates, this object is relatively close to the Sun, and hence obtaining a parallax measurement should be relatively easy. Provided this object is indeed an sdT0 dwarf, the discovery of WISE J0711-5736 would only be the second low-metallicity late-L/early-T dwarf discovered to date. Further spectroscopic observations will allow us to be able to definitively determine the metallicity and thus, the membership, of this object. New observations will also allow us to rule out (or confirm) multiplicity.

We thank the anonymous referees for their very constructive comments that helped improve this paper. K.K. acknowledges support via the IPAC Visiting Graduate Student Program. This publication makes use of data products from the Wide-field Infrared Survey Explorer, which is a joint project of the University of California, Los Angeles, and the Jet Propulsion Laboratory/California Institute of Technology, funded by the National Aeronautics and Space Administration.

Facility: Magellan: Baade (FIRE).

Software: IDL.

\section{ORCID iDs}

Kendra Kellogg (iD https://orcid.org/0000-0002-1819-3387 Stanimir Metchev (iD https://orcid.org/0000-0003-3050-8203 Jonathan Gagné (i) https://orcid.org/0000-0002-2592-9612 Jacqueline K. Faherty (iD https://orcid.org/0000-00016251-0573

\section{References}

Ackerman, A. S., \& Marley, M. S. 2001, ApJ, 556, 872

Baraffe, I., Homeier, D., Allard, F., \& Chabrier, G. 2015, A\&A, 577, A42 Bardalez Gagliuffi, D. C., Gelino, C. R., \& Burgasser, A. J. 2015, AJ, 150, 163 Bochanski, J. J., Hennawi, J. F., Simcoe, R. A., et al. 2009, PASP, 121, 1409 Burgasser, A. J. 2004, ApJS, 155, 191

Burgasser, A. J., Cruz, K. L., Cushing, M., et al. 2010, ApJ, 710, 1142

Burgasser, A. J., Geballe, T. R., Leggett, S. K., Kirkpatrick, J. D., \& Golimowski, D. A. 2006, ApJ, 637, 1067

Burgasser, A. J., Kirkpatrick, J. D., Brown, M. E., et al. 1999, ApJL, 522, L65 Burgasser, A. J., Kirkpatrick, J. D., Burrows, A., et al. 2003, ApJ, 592, 1186 
Burgasser, A. J., Liu, M. C., Ireland, M. J., Cruz, K. L., \& Dupuy, T. J. 2008a, ApJ, 681, 579

Burgasser, A. J., Looper, D. L., Kirkpatrick, J. D., Cruz, K. L., \& Swift, B. J. 2008b, ApJ, 674, 451

Burgasser, A. J., Marley, M. S., Ackerman, A. S., et al. 2002, ApJL, 571, L151

Burgasser, A. J., McElwain, M. W., Kirkpatrick, J. D., et al. 2004, AJ, 127, 2856

Burningham, B., Smith, L., Cardoso, C. V., et al. 2014, MNRAS, 440, 359

Burrows, A., Hubbard, W. B., Lunine, J. I., \& Liebert, J. 2001, RvMP, 73, 719

Burrows, A., Hubbard, W. B., Saumon, D., \& Lunine, J. I. 1993, ApJ, 406, 158

Burrows, A., Sudarsky, D., \& Hubeny, I. 2006, ApJ, 640, 1063

Chabrier, G., \& Baraffe, I. 1997, A\&A, 327, 1039

Chiba, M., \& Beers, T. C. 2000, AJ, 119, 2843

Chiu, K., Fan, X., Leggett, S. K., et al. 2006, AJ, 131, 2722

Cruz, K. L., Reid, I. N., Kirkpatrick, J. D., et al. 2007, AJ, 133, 439

Cushing, M. C., Vacca, W. D., \& Rayner, J. T. 2004, PASP, 116, 362

Dieterich, S. B., Henry, T. J., Jao, W.-C., et al. 2014, AJ, 147, 94

Dupuy, T. J., \& Liu, M. C. 2012, ApJS, 201, 19

Dupuy, T. J., \& Liu, M. C. 2017, ApJS, 231, 15

Faherty, J. K., Riedel, A. R., Cruz, K. L., et al. 2016, ApJS, 225, 10

Feltzing, S., Bensby, T., \& Lundström, I. 2003, A\&A, 397, L1

Filippazzo, J. C., Rice, E. L., Faherty, J., et al. 2015, ApJ, 810, 158

Fuhrmann, K. 1998, A\&A, 338, 161

Gagné, J., Faherty, J. K., Cruz, K. L., et al. 2015, ApJS, 219, 33

Gagné, J., Lambrides, E., Faherty, J. K., \& Simcoe, R. 2015, Firehose v2.0, Zenodo, doi:10.5281/zenodo.18775

Gazzano, J.-C., Kordopatis, G., Deleuil, M., et al. 2013, A\&A, 550, A125

Kirkpatrick, J. D. 2005, ARA\&A, 43, 195
Kirkpatrick, J. D., Cushing, M. C., Gelino, C. R., et al. 2011, ApJS, 197, 19 Kirkpatrick, J. D., Kellogg, K., Schneider, A. C., et al. 2016, ApJS, 224, 36 Kirkpatrick, J. D., Looper, D. L., Burgasser, A. J., et al. 2010, ApJS, 190, 100 Kirkpatrick, J. D., Schneider, A., Fajardo-Acosta, S., et al. 2014, ApJ, 783, 122 Lépine, S., Rich, R. M., \& Shara, M. M. 2007, ApJ, 669, 1235

Liu, M. C., Leggett, S. K., Golimowski, D. A., et al. 2006, ApJ, 647, 1393 Luhman, K. L. 2014, ApJL, 786, L18

Luhman, K. L., \& Sheppard, S. S. 2014, ApJ, 787, 126

Mace, G. N., Kirkpatrick, J. D., Cushing, M. C., et al. 2013a, ApJS, 205, 6

Mace, G. N., Kirkpatrick, J. D., Cushing, M. C., et al. 2013b, ApJ, 777, 36

Mainzer, A., Bauer, J., Grav, T., et al. 2011, ApJ, 731, 53

Marley, M. S., Saumon, D., Guillot, T., et al. 1996, Sci, 272, 1919

Marley, M. S., Seager, S., Saumon, D., et al. 2002, ApJ, 568, 335

Pinfield, D. J., Gomes, J., Day-Jones, A. C., et al. 2014, MNRAS, 437, 1009

Radigan, J., Jayawardhana, R., Lafrenière, D., et al. 2013, ApJ, 778, 36

Reddy, B. E., Lambert, D. L., \& Allende Prieto, C. 2006, MNRAS, 367, 1329

Schilbach, E., Röser, S., \& Scholz, R.-D. 2009, A\&A, 493, L27

Schmidt, S. J., West, A. A., Hawley, S. L., \& Pineda, J. S. 2010, AJ, 139, 1808

Simcoe, R. A., Burgasser, A. J., Bernstein, R. A., et al. 2008, Proc. SPIE, 7014, $70140 \mathrm{U}$

Simcoe, R. A., Burgasser, A. J., Schechter, P. L., et al. 2013, PASP, 125, 270

Sivarani, T., Lépine, S., Kembhavi, A. K., \& Gupchup, J. 2009, ApJL, 694, L140

Soubiran, C., Bienaymé, O., \& Siebert, A. 2003, A\&A, 398, 141

Zhang, Z. H., Homeier, D., Pinfield, D. J., et al. 2017a, MNRAS, 468, 261

Zhang, Z. H., Pinfield, D. J., Gálvez-Ortiz, M. C., et al. 2017b, MNRAS, 464,3040 